\title{
Caffeine Consumption Contributes to Skin Intrinsic Fluorescence in Type 1 Diabetes
}

\author{
Karen M. Eny, PhD, Trevor J. Orchard, MD, Rachel Grace Miller, MS, ${ }^{2}$ John Maynard, MSEE, \\ Denis M. Grant, PhD, Tina Costacou, PhD, ${ }^{2}$ Patricia A. Cleary, MS, ${ }^{5}$ Barbara H. Braffett, PhD, \\ the DCCT/EDIC Research Group, ${ }^{*}$ and Andrew D. Paterson, MD ${ }^{1,6}$
}

\begin{abstract}
Background: A variant (rs1495741) in the gene for the $N$-acetyltransferase 2 (NAT2) protein is associated with skin intrinsic fluorescence (SIF), a noninvasive measure of advanced glycation end products and other fluorophores in the skin. Because NAT2 is involved in caffeine metabolism, we aimed to determine whether caffeine consumption is associated with SIF and whether rs1495741 is associated with SIF independently of caffeine. Materials and Methods: SIF was measured in 1,181 participants with type 1 diabetes from the Epidemiology of Diabetes Interventions and Complications study. Two measures of SIF were used: SIF1, using a 375-nm excitation light-emitting diode (LED), and SIF14 (456-nm LED). Food frequency questionnaires were used to estimate mean caffeine intake. To establish replication, we examined a second type 1 diabetes cohort.

Results: Higher caffeine intake was significantly associated with higher $\operatorname{SIF}_{\text {LED }} 375 \mathrm{~nm}[0.6,0.2]\left(P=2 \times 10^{-32}\right)$ and SIF14 LED $456 \mathrm{~nm}[0.4,0.8]_{(}\left(P=7 \times 10^{-31}\right)$ and accounted for $4 \%$ of the variance in each after adjusting for covariates. When analyzed together, caffeine intake and rs1495741 both remained highly significantly associated with SIF1 ${ }_{\text {LED }} 375 \mathrm{~nm}[0.6,0.2]$ and SIF14 ${ }_{\text {LED } 456 \mathrm{~nm}[0.4,0.8]}$. Mean caffeinated coffee intake was also positively associated with SIF1 $1_{\text {LED }} 375 \mathrm{~nm}[0.6,0.2]\left(P=9 \times 10^{-12}\right)$ and $\operatorname{SIF} 14_{\text {LED } 456 \mathrm{~nm}[0.4,0.8]}\left(P=4 \times 10^{-12}\right)$, but no association was observed for decaffeinated coffee intake. Finally, caffeine was also positively associated with SIF1 $1_{\text {LED }} 375 \mathrm{~nm}[0.6,0.2]$ and SIF14 ${ }_{\text {LED }} 456 \mathrm{~nm}[0.4,0.8](P<0.0001)$ in the replication cohort.

Conclusions: Caffeine contributes to SIF. The effect of rs1495741 on SIF appears to be partially independent of caffeine consumption. Because SIF and coffee intake are each associated with cardiovascular disease, our findings suggest that accounting for coffee and/or caffeine intake may improve risk prediction models for SIF and cardiovascular disease in individuals with diabetes.
\end{abstract}

\section{Introduction}

$\mathbf{M}$ EASUREMENT OF SKIN INTRINSIC fluorescence (SIF) represents a novel noninvasive biomarker of advanced glycation end products (AGEs) and other fluor- ophores in the skin. ${ }^{1,2}$ Skin fluorescence has been shown to be positively associated with subclinical atherosclerosis and/or cardiovascular disease mortality in the general population, ${ }^{3}$ in type 1 and type 2 diabetes, ${ }^{4-6}$ and in people with renal failure. ${ }^{7}$

\footnotetext{
${ }^{1}$ Program in Genetics and Genome Biology, Hospital for Sick Children, Toronto, Ontario, Canada.

${ }^{2}$ Department of Epidemiology, University of Pittsburgh, Pittsburgh, Pennsylvania.

${ }^{3}$ VeraLight, Inc., Albuquerque, New Mexico.

${ }^{4}$ Department of Pharmacology \& Toxicology, University of Toronto, Toronto, Ontario, Canada.

${ }^{5}$ The Biostatistics Center, The George Washington University, Rockville, Maryland.

${ }^{6}$ Dalla Lana School of Public Health, University of Toronto, Toronto, Ontario, Canada.

*A complete list of participants in the DCCT/EDIC Research Group is presented in the Supplementary Material published online for the article in N Engl J Med 2015;372:1722-1733 and is given in Supplementary Data (available online at www.liebertonline.com/dia).

The DCCT and the EDIC are registered at ClinicalTrials.gov with clinical trial registration numbers NCT00360815 and NCT00360893, respectively.

The content of this article is solely the responsibility of the authors and does not necessarily reflect the official views of the National Institute of Diabetes and Digestive and Kidney Diseases.

(C) The Author(s) 2015; Published by Mary Ann Liebert, Inc. This Open Access article is distributed under the terms of the Creative Commons Attribution Noncommercial License (http://creativecommons.org/licenses/by-nc/4.0/) which permits any noncommercial use, distribution, and reproduction in any medium, provided the original author(s) and the source are credited.
} 
Several factors contribute to interindividual variability in skin fluorescence, including age, glycated hemoglobin (HbAlc), smoking and nephropathy. ${ }^{7,8}$ Recently, rs1495741 in the gene for the $N$-acetyltransferase 2 (NAT2) protein, which tags arylamine $N$-acetyltransferase enzyme activity status, ${ }^{9}$ was identified as a major locus influencing skin fluorescence across four cohorts consisting of subjects with and without diabetes, explaining up to $15 \%$ of the variance. ${ }^{10}$ NAT2 is a phase II drugmetabolizing enzyme that metabolizes aromatic amine and hydrazine drugs, heterocyclic amines, ${ }^{11}$ and caffeine following initial detoxification by CYP1A2. ${ }^{12}$ NAT2 has been previously associated with serum 1-methylxanthine:4-acetamidobutanoate $\left(P=1.7 \times 10^{-40}\right)$ in a genome-wide association study (GWAS). ${ }^{13}$ This metabolite ratio likely reflects the role of NAT2 in the metabolism of caffeine because caffeine is metabolized to 1-methylxanthine, which is used to determine the NAT2 acetylator status. ${ }^{12}$ Because caffeine exhibits fluorescent properties ${ }^{14}$ and NAT2 metabolizes caffeine metabolites, it is plausible that the NAT2 association with SIF is due to fluorescence of caffeine in the skin. We therefore tested whether caffeine consumption is associated with SIF and whether the association of rs1495741 with SIF is independent of caffeine intake. We separately used two cohorts of subjects with type 1 diabetes to determine the reproducibility of our observations.

\section{Materials and Methods}

\section{Subjects}

Subjects included 1,441 patients with type 1 diabetes between 13 and 39 years of age at baseline, who were recruited from 1983 to 1989 to participate in the Diabetes Control and Complications Trial (DCCT). ${ }^{15}$ Participants were randomized to intensive $(n=711)$ or conventional $(n=730)$ therapy and were followed up for a mean of 6.5 years until the study ended in 1993. In 1994, 1,375 subjects ( $96 \%$ of the surviving cohort) were enrolled in the annual observational follow-up study, Epidemiology of Diabetes Interventions and Complications (EDIC). ${ }^{16}$ In EDIC year 16 or 17, subjects were invited to participate in the SCOUT substudy to measure SIF, and $92 \%(n=1,185)$ of the active EDIC subjects participated. ${ }^{8}$

\section{Measurement of SIF}

The SCOUT DS ${ }^{\circledR}$ SF spectrometer (VeraLight, Inc., Albuquerque, NM) was used to measure skin tone (pigmentation) and SIF from the underside of the left forearm. SIF is a measure of skin fluorescence that mathematically corrects for factors such as skin pigmentation, which may affect light absorption and scattering. ${ }^{4,8,17}$ For all of our analyses we used SIF1 $1_{\text {LED } 375 \mathrm{~nm}[0.6,0.2]}$ and SIF14 LED 456nm[0.4,0.8], which were examined previously in genetic association studies of SIF in DCCT/EDIC $^{10}$ and represent the lowest $(\mathrm{SIF} 1=375 \mathrm{~nm})$ and highest (SIF14 $=456 \mathrm{~nm}$ ) light-emitting diode (LED) excitation wavelengths examined in the DCCT/EDIC study. Furthermore, SIF1 LED $375 \mathrm{~nm}[0.6,0.2]$ as measured by the SCOUT DS SF spectrometer is similar to the excitation level (approximately $350-370 \mathrm{~nm}$ ) commonly used by the AGE Reader (DiagnOptics, Groningen, The Netherlands), another device that measures skin fluorescence., 3,7 This level of excitation captures fluorescence of AGEs and has been pre- viously shown to have the strongest association with type 1 diabetes complications in the Pittsburgh Epidemiology of Diabetes Complications (EDC) study. ${ }^{1,2,5,18}$

SIF1 LED $375 \mathrm{~nm}[0.6,0.2]$ was detected over an emission range of $435-655 \mathrm{~nm}$ and corrected for light scattering with the reflectance adjusted by the dimensionless excitation and emission exponents, $k_{x}=0.6$ and $k_{m}=0.2$, respectively. SIF14 1 LED $456 \mathrm{~nm}[0.4,0.8]$ was detected over an emission range of $491-655 \mathrm{~nm}$, with reflectance adjusted by $k_{x}=0.4$ and $k_{m}=0.8$ emission exponents. The first of two SIF measurements, reported in arbitrary units, was used for analyses.

\section{Measurement of caffeine intake}

During the DCCT (1983-1993), diet was assessed using a modified Burke-type diet history ${ }^{19}$ at the baseline, 2-year, 5year, and study end points. Participants were asked by dietitians to describe the meals and snacks they usually had to eat or drink on weekdays and on weekends. Seasonal variations in food intake and foods eaten away from home were also recorded. A staff of trained coders at the Central Nutrition Coding Unit (University of Minnesota, St. Paul, MN) coded the diet histories in a standardized manner, and nutrient intakes were calculated using version 13 (1986) of the Nutrition Coordinating Center nutrient database. The intercoder reliability and the reproducibility of the diet history were assessed at Year 2 of the DCCT. ${ }^{19}$

During EDIC years 1-12 (1994-2006), a 126-item selfadministered Harvard food frequency questionnaire (FFQ) was used to assess diet intake biennially. ${ }^{20}$ Participants were asked how often they consumed a specified serving size of each food item over the past year. The nine response categories ranged from never or less than once per month to six or more times daily. Nutrient intakes from the FFQ were then calculated to reflect daily intake using the corresponding food item and respective portion size primarily from the U.S. Department of Agriculture nutrient database. Frequency response items were available for caffeinated beverages (coffee, tea, cola, and low-calorie cola) and decaffeinated coffee during EDIC Years 13-15. The validity of the Harvard FFQ was assessed previously in a subset of participants from the Nurses' Health Study by comparing the FFQ with 1-week diet records measured approximately every 3 months during the preceding year. Correlation coefficients for coffee and tea consumption were 0.75 and 0.90 , respectively. ${ }^{21}$

\section{Genotyping}

The rs1495741 (NAT2) genotype was available from the Illumina (San Diego, CA) human $1 \mathrm{M}$ beadchip assay, which was used for genome-wide genotyping in the DCCT/EDIC. ${ }^{10}$ After exclusion of subjects determined to be admixed using population genetic approaches, 1,081 subjects had both rs1495741 genotype data and SIF measurements available. ${ }^{10}$

\section{Statistical analysis}

Caffeine intake measured using the FFQ during the EDIC study was used as our primary measure of caffeine exposure. Over $60 \%$ of subjects had four or five measures of caffeine intake from FFQs completed during the EDIC study (range, one to seven), and approximately $50 \%$ had three measures of caffeine intake from diet histories completed during the 
DCCT (range, one to six). Mean caffeine intake was calculated separately for each subject during the DCCT and the EDIC study. To approximate a normal distribution, mean caffeine intake was square root transformed when used as the outcome variable (Supplementary Figs S1 and S2; Supplementary Data are available online at www.liebertonline.com/ dia), whereas SIF1 $1_{\text {LED } 375 \mathrm{~nm}[0.6,0.2]}$ and SIF14 ${ }_{\text {LED } 456 \mathrm{~nm}[0.4,0.8]}$ were $\log _{e}$ transformed. ${ }^{10}$

rs1495741 was modeled assuming an additive model. Linear regression was used to examine the relationship of rs1495741 with SIF1 $1_{\text {LED } 375 \mathrm{~nm}[0.6,0.2]}$ and SIF14 ${ }_{\text {LED } 456 \mathrm{~nm}[0.4,0.8]}$ in both unadjusted (M1) and adjusted (M2) models. M2 included age, sex, skin tone, EDIC clinic latitude, smoking status, any estimated glomerular filtration rate $<60 \mathrm{~mL} / \mathrm{min} /$ $1.73 \mathrm{~m}^{2}$, DCCT eligibility HbA1c, mean DCCT HbA1c, and mean EDIC HbA1c ${ }^{8}$ (Supplementary Table S1). The same models were also used to examine the association of mean caffeine intake during the EDIC study and/or the DCCT alone or in combination with SIF. To exclude the possibility of confounding due to total reported daily caloric intake, it was included as a covariate in secondary models after excluding under- and over-reporters, defined as consuming $<800 \mathrm{kcal} /$ day and $>6,000 \mathrm{kcal} /$ day, respectively. We also additionally adjusted M2 for DCCT treatment group and separately for DCCT prevention cohorts in secondary analyses. To examine whether a nonlinear relationship exists between caffeine intake and SIF, caffeine ${ }^{2}$ was added to M1 and M2. Linear regression was used to test whether rs 1495741 was associated with caffeine intake. To determine whether the association of rs1495741 with SIF differed by smoking status, we tested for interaction between rs 1495741 and smoking status (never smokers vs. ever and current smokers).

Unadjusted Spearman correlation, and multiple linear regression, adjusting for M2 covariates, were used to determine the association of mean consumption of each of the caffeinated beverages and decaffeinated coffee frequency with SIF. A value of $P<0.05$ was used to establish statistical significance.

\section{Replication study}

Subjects comprised 210 participants (48\% male) from the Pittsburgh EDC study of childhood-onset diabetes ${ }^{22}$ who had caffeine intake and SIF measured. Diet was assessed at the baseline (1986-1988), 2-year, and 10-year exams using the same Harvard FFQ used in the EDIC study. Mean caffeine intake was calculated for each person and used for analyses.

SIF was initially measured in a pilot group of participants living within 25 miles of the study clinic in 2007-2009 $(n=107)$ and subsequently in a substudy of participants undergoing brain imaging in $2010(n=65)$ and at the 25-year examination in $2011(n=38)$. By June 30, 2013, a total of 210 subjects had SIF measured and were included in the SIFcaffeine analyses. For participants with two SIF measurements available, only the first measurement was used for analyses. On average, SIF was assessed 17.9 \pm 3.0 (SD) years (range, 8.7-26.5 years) after diet was assessed, using a similar protocol as in the DCCT/EDIC study. ${ }^{5}$

DNA was collected during the 2-year exam, and rs1495741 was genotyped using fluorescence polarization with a $5^{\prime}$-ctatcttccagaaagtaaatgtg- $3^{\prime}$ forward primer, $5^{\prime}$-tggga aactatcatttaaaacag- $3^{\prime}$ reverse primer, and 5-gaagctactgtgaat gccca- $3^{\prime}$ fluorescence polarization reverse primer and detected on $2 \%$ agarose gel. Polymerase chain reaction conditions were $95^{\circ} \mathrm{C}$ for $5 \mathrm{~min}, 35$ cycles of $\left(95^{\circ} \mathrm{C}\right.$ for $30 \mathrm{~s}, 56^{\circ} \mathrm{C}$ for $30 \mathrm{~s}$, and $72^{\circ} \mathrm{C}$ for $30 \mathrm{~s}$ ), and finally $72^{\circ} \mathrm{C}$ for $5 \mathrm{~min}$ and $10^{\circ} \mathrm{C}$ hold. For fluorescence polarization an annealing temperature of $60^{\circ} \mathrm{C}$ using the $\mathrm{C} / \mathrm{T}$ dye mix was used.

Multiple linear regression adjusting for age, sex, smoking, and estimated glomerular filtration rate $<60 \mathrm{~mL} / \mathrm{min} / 1.73 \mathrm{~m}^{2}$ was used to test for association between rs 1495741 with SIF. Spearman correlations were used to examine the relationship for mean caffeine intake with SIF. Multiple linear regression was used to test the joint effects of rs1495741 and mean caffeine intake on SIF including age, sex, smoking, and estimated glomerular filtration rate $<60 \mathrm{~mL} / \mathrm{min} / 1.73 \mathrm{~m}^{2}$ as additional covariates.

\section{Results}

Table 1 shows subject characteristics of the DCCT/EDIC participants, separately by the former DCCT treatment group. On average, daily caffeine intake during the EDIC study (Supplementary Fig. S1) was $74 \mathrm{mg} /$ day lower than during the DCCT (Supplementary Fig. S2), but caffeine intake was correlated across DCCT and EDIC time periods $(r=0.74$, $P<0.0001)$.

Mean caffeine intake during the EDIC study was positively associated with SIF1 $1_{\text {LED } 375 \mathrm{~nm}[0.6,0.2]}$ and SIF14 ${ }_{\text {LED } 456 \mathrm{~nm}[0.4,0.8]}$ in unadjusted analyses and accounted for approximately $11 \%$ of the variance in each, respectively (Table 2 and Supplementary Fig. S3).

After adjusting for covariates, caffeine was still significantly associated with SIF1 $1_{\text {LED } 375 \mathrm{~nm}[0.6,0.2]}$ and SIF14 ${ }_{\text {LED } 456 \mathrm{~nm}[0.4,0.8]}$, but the effect was attenuated, and the variance accounted for by caffeine intake during the EDIC study was $3.8 \%$ for $\mathrm{SIF}_{\text {LED }} 375 \mathrm{~nm}[0.6,0.2]$ and $4.2 \%$ for SIF14 ${ }_{\text {LED }} 456 \mathrm{~nm}[0.4,0.8]$ (Table 2).

Because age, smoking status, and mean EDIC HbA1c were all positively associated with both $\operatorname{SIF} 1_{\text {LED }} 375 \mathrm{~nm}[0.6,0.2]$ (Supplementary Table S1) and mean EDIC caffeine intake (Supplementary Table S2), we examined whether the attenuation of the caffeine effect on SIF in M2 (Table 2) was explained by adjusting for these variables. Adjusting for age and smoking status largely accounted for the attenuation of the caffeine effect on $\mathrm{SIF}_{\text {LED } 375 \mathrm{~nm}[0.6,0.2]}(\beta \pm \mathrm{SE}, 0.00031 \pm$ $0.000025 ; P=1.9 \times 10^{-33}$, without adjustments for age and smoking status). Similarly, the attenuation of the caffeine effect on SIF14 LED $456 \mathrm{~nm}[0.4,0.8]$ Was due to adjustments for age and smoking status as well as EDIC HbA1c $(\beta \pm \mathrm{SE}, 0.00035 \pm$ $0.00003 ; P=1.6 \times 10^{-30}$, without adjustments for age, smoking status, and $\mathrm{HbA1c}$ ).

Additionally adjusting M2 for mean total caloric intake did not materially alter the association of mean EDIC caffeine intake with either $\operatorname{SIF}_{\text {LED } 375 \mathrm{~nm}[0.6,0.2]}\left(P=4.1 \times 10^{-17}\right)$ or SIF14 ${ }_{\text {LED }} 456 \mathrm{~nm}[0.4,0.8]\left(P=3.9 \times 10^{-16}\right)$. Similarly, adjusting for DCCT treatment group or DCCT primary versus secondary prevention cohort did not materially alter the results (data not shown).

We also tested whether the association of caffeine intake with SIF is nonlinear and observed some evidence supporting a quadratic relationship for $\mathrm{SIF} 1_{\mathrm{LED}} 375 \mathrm{~nm}[0.6,0.2]\left(\beta_{\text {linear }} \pm\right.$ $\mathrm{SE}, 0.0002 \pm 0.00003\left[P=5.8 \times 10^{-16}\right] ; \beta_{\text {quadratic }} \pm \mathrm{SE},-1.8 \times$ 
Table 1. Characteristics of the Diabetes Control and Complications Trial (DCCT)/Epidemiology of Diabetes Interventions and Complications Participants with Skin Instrinsic Fluorescence (SIF) Measures, Separately by Former DCCT Randomized Treatment Group at the Time Sif Was Measured

\begin{tabular}{|c|c|c|}
\hline & Former INT $(\mathrm{n}=612)$ & Former $\operatorname{CON}(\mathrm{n}=573)$ \\
\hline \multicolumn{3}{|l|}{ Demographic characteristics } \\
\hline Male sex & $317(52 \%)$ & $308(54 \%)$ \\
\hline Age (years) & $52 \pm 7$ & $51 \pm 7$ \\
\hline Diabetes duration (years) & $30.0 \pm 4.9$ & $29.5 \pm 4.9$ \\
\hline Primary cohort assignment ${ }^{\mathrm{a}}$ & $298(49 \%)$ & $294(51 \%)$ \\
\hline Skin tone (arbitrary units) & $260 \pm 47$ & $256 \pm 49$ \\
\hline Clinic latitude $\left(>37^{\circ} \mathrm{N}\right)^{\mathrm{b}}$ & $444(73 \%)$ & $427(75 \%)$ \\
\hline \multicolumn{3}{|l|}{ Smoking status ${ }^{\mathrm{c}}$} \\
\hline Never & $372(61 \%)$ & $352(61 \%)$ \\
\hline Former & $154(25 \%)$ & $149(26 \%)$ \\
\hline Current & $86(14 \%)$ & $72(13 \%)$ \\
\hline Any eGFR $<60 \mathrm{~mL} / \mathrm{min} / 1.73 \mathrm{~m}^{2}$ to date $(y e s)^{\mathrm{d}}$ & $40(7 \%)$ & $44(8 \%)$ \\
\hline \multicolumn{3}{|l|}{ Glycemic exposure } \\
\hline DCCT eligibility HbA1c (\%) (mmol/mol) & $9.1 \pm 1.6(76 \pm 17)$ & $8.9 \pm 1.6(74 \pm 17)$ \\
\hline DCCT mean HbAlc (\%) (mmol/mol) & $7.2 \pm 0.8(55 \pm 9)$ & $9.0 \pm 1.3(75 \pm 14)$ \\
\hline EDIC mean $\mathrm{HbAlc}(\%)(\mathrm{mmol} / \mathrm{mol})$ & $8.0 \pm 1.1(63 \pm 12)$ & $8.0 \pm 1.0(63 \pm 11)$ \\
\hline Time-weighted mean $\mathrm{HbAlc}(\%)(\mathrm{mmol} / \mathrm{mol})^{\mathrm{e}}$ & $8.0 \pm 0.9(64 \pm 10)$ & $8.4 \pm 0.9(68 \pm 10)$ \\
\hline Mean caffeine intake during DCCT (mg/day) & $369 \pm 329$ & $369 \pm 342$ \\
\hline Mean caffeine intake during EDIC $(\mathrm{mg} / \mathrm{d})^{\mathrm{f}}$ & $301 \pm 218$ & $288 \pm 209$ \\
\hline Time between caffeine intake assessment and SIF1 (years) ${ }^{\mathrm{g}}$ & $11.1 \pm 1.1$ & $11.0 \pm 1.0$ \\
\hline rs1495741 genotype $(\mathrm{AA} / \mathrm{AG} / \mathrm{GG})^{\mathrm{h}}$ & $342 / 189 / 24$ & $307 / 191 / 28$ \\
\hline SIF1 $_{\text {LED }} 375 \mathrm{~nm}, k x=0.6, k m=0.2$ (arbitrary units) ${ }^{\mathrm{i}}$. & $3.1 \pm 0.2$ & $3.1 \pm 0.21$ \\
\hline SIF14 $_{\text {LED }} 456 \mathrm{~nm}, k x=0.4, k m=0.8$ (arbitrary units) ${ }^{\mathrm{i}}$ & $0.37 \pm 0.23$ & $0.36 \pm 0.23$ \\
\hline
\end{tabular}

Data are $n(\%)$ or mean \pm SD values as indicated $(n=1,185)$.

${ }^{\mathrm{a}}$ Two cohorts were recruited at DCCT baseline: a primary cohort $(n=726)$ of subjects with no retinopathy and a urinary albumin excretion rate of $<40 \mathrm{mg} / 24 \mathrm{~h}$ at baseline and a secondary cohort $(n=715)$ of subjects exhibiting mild to moderate nonproliferative retinopathy and urinary albumin excretion rate of $\leq 200 \mathrm{mg} / 24 \mathrm{~h}$ at baseline.

${ }^{\mathrm{b}} \mathrm{Clinic}$ latitude was categorized as a binary variable with clinics located above $37^{\circ} \mathrm{N}$ latitude designated as northern clinics $(n=21)$ and those below assigned as southern clinics $(n=7)$.

"Smoking status was defined as "never smoker" ( $\leq 100$ cigarettes in a subject's lifetime), "former smoker" (quit $\geq 1$ year ago), or "current smoker" (currently smoking or smoking within the last year).

${ }^{\mathrm{d}}$ Estimated glomerular filtration rate (eGFR) was estimated using the Chronic Kidney Disease-Epidemiology Collaboration equation.

${ }^{\mathrm{e}}$ Time-weighted mean glycated hemoglobin $(\mathrm{HbAlc})$ is calculated by summing (DCCT eligibility HbAlc $\times$ duration of diabetes at DCCT baseline), (DCCT mean HbA1c $\times$ years of follow-up in DCCT), and (Epidemiology of Diabetes Interventions and Complications [EDIC] mean $\mathrm{HbA} 1 \mathrm{c} \times$ years of follow-up in EDIC) and dividing by total duration of diabetes.

${ }^{\mathrm{f}}$ Two subjects in the intensive treatment (INT) group and two in the conventional treatment (CON) group did not have dietary caffeine intake available.

${ }^{\mathrm{g}}$ The minimum lag time between measures of caffeine intake during EDIC and measures of SIF was 4 years.

${ }^{\mathrm{h}}$ One hundred four subjects did not have rs1495741 genotype data available.

${ }^{\mathrm{i}} \mathrm{Ln}$ transformed.

LED, light-emitting diode.

Table 2. Association of Caffeine Intake During the Epidemiology of Diabetes Interventions and Complications Study with Skin Intrinsic Fluorescence

\begin{tabular}{lccc}
\hline SIF outcome (excitation wavelength), model & Variance & $\beta \pm S E$ & P value \\
\hline SIF1 (375 nm) $k x=0.6, k m=0.2^{a}$ & & & \\
Unadjusted (M1) & $11.2 \%$ & $0.000321 \pm 2.63 \mathrm{E}-05$ & $2.28 \times 10^{-32}$ \\
$\quad$ Adjusted (M2) & $3.8 \%$ & $0.000203 \pm 2.43 \mathrm{E}-05$ & $1.66 \times 10^{-16}$ \\
SIF14 (456 nm) $k x=0.4, k m=0.8^{\mathrm{a}}$ & & & $7.19 \times 10^{-31}$ \\
$\quad$ Unadjusted (M1) & $10.7 \%$ & $0.000356 \pm 3 \mathrm{E}-05$ & $7.19 \times 10^{-16}$ \\
$\quad$ Adjusted (M2) & $4.2 \%$ & $0.000244 \pm 2.98 \mathrm{E}-05$ & \\
\hline
\end{tabular}

The value of $\beta \pm$ SE was obtained from linear regression $(n=1,181)$. Variance was calculated as a type II squared semipartial correlation. Adjusted models included age, sex, skin tone, clinic latitude, smoking status, any estimated glomerular filtration rate of $<60 \mathrm{~mL} / \mathrm{min} /$ $1.73 \mathrm{~m}^{2}$, Diabetes Control and Complications Trial eligibility hemoglobinA1c, mean Diabetes Control and Complications Trial hemoglobin A1c, and mean Epidemiology of Diabetes Interventions and Complications hemoglobin A1c as covariates.

${ }^{a}$ Ln transformed.

M1, Model 1; M2, Model 2; SIF, skin intrinsic fluorescence. 
$\left.10^{-7} \pm 8 \times 10^{-8}[P=0.03]\right)$ and SIF14 ${ }_{\text {LED } 456 \mathrm{~nm}[0.4,0.8]}\left(\beta_{\text {linear }} \pm\right.$ SE, $0.0003 \pm 0.00003\left[P=1.0 \times 10^{-16}\right] ; \beta_{\text {quadratic }} \pm \mathrm{SE},-1.9 \times$ $\left.10^{-7} \pm 1 \times 10^{-7}[P=0.05]\right)$.

Caffeine intake during DCCT was consistently positively associated with SIF1 $1_{\text {LED }} 375 \mathrm{~nm}[0.6,0.2]$ and SIF14 4 LED $456 \mathrm{~nm}[0.4,0.8]$, independently accounting for $2.7 \%$ and $2.3 \%$ of the variance, respectively, after adjusting for covariates in M2 (Supplementary Table S3). Mean caffeine intakes from both the DCCT and the EDIC study were independently associated with $\mathrm{SIF}_{\mathrm{LED}}$ $375 \mathrm{~nm}[0.6,0.2]$ and SIF14 LED $456 \mathrm{~nm}[0.4,0.8]$ when included in the model together, with the exception for the association of mean caffeine intake during DCCT with SIF14 ${ }_{\text {LED } 456 \mathrm{~nm}[0.4,0.8]}$ in M2 (Supplementary Table S4).

As reported previously, ${ }^{10}$ rs1495741 was significantly associated with $\mathrm{SIF}_{\mathrm{LED}} 375 \mathrm{~nm}[0.6,0.2](\beta \pm \mathrm{SE},-0.065 \pm 0.010$; $\left.P=6.1 \times 10^{-10}\right)$ and SIF14 ${ }_{\text {LED }} 456 \mathrm{~nm}[0.4,0.8](\beta \pm \mathrm{SE},-0.125 \pm$ $\left.0.011 ; P=8.06 \times 10^{-27}\right)$. The results were even stronger after

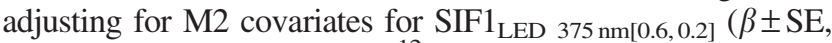
$\left.-0.060 \pm 0.008 ; P=1.7 \times 10^{-12}\right)$ and SIF14 $\mathrm{LED} 456 \mathrm{~nm}[0.4,0.8$ $\left(\beta \pm \mathrm{SE},-0.123 \pm 0.0096 ; P=1.4 \times 10^{-34}\right)$. Each copy of the $\mathrm{G}$ allele was associated with lower SIF, explaining $3.5 \%$ and $10.1 \%$ of the variance in SIF1 $1_{\mathrm{LED}} 375 \mathrm{~nm}[0.6,0.2]$ and SIF14 4 LED $456 \mathrm{~nm}[0.4,0.8]$, respectively. ${ }^{10}$ Because NAT2 metabolizes aromatic amines found in tobacco smoke ${ }^{11}$ and because smoking status is an important confounder, we tested whether the NAT2 (rs1495741) effect was modified by smoking status. However, there was no significant interaction of rs1495741 with smoking status on SIF1 $1_{\text {LED }} 375 \mathrm{~nm}[0.6,0.2](P=0.13)^{10}$ or SIF14 ${ }_{\text {LED }} 456 \mathrm{~nm}[0.4,0.8](P=0.39)$.

Prior to determining the joint effects of rs1495741 and mean caffeine intake during the EDIC study on SIF, we showed that rs1495741 was not associated with mean caffeine intake either during the EDIC study $(P=0.51)$ or the DCCT $(P=0.42)$. Then we showed that models containing both rs1495741 and caffeine intake during EDIC were independently associated with SIF $1_{\text {LED } 375 \mathrm{~nm}[0.6,0.2]}$ and SIF14 LED $456 \mathrm{~nm}[0.4,0.8]$ in fully adjusted analyses (Table 3 ).

To determine whether the associations for caffeine intake on SIF are due to caffeine or other constituents found in coffee, which may correlate with caffeine intake, we tested whether frequency of coffee, decaffeinated coffee, tea, and caffeinated cola (regular and low-calorie) consumption was associated with SIF. Supplementary Table S5 shows the number of subjects reporting a mean consumption of at least once per month for each beverage during EDIC years 13-15. In both Spearman correlation $\left(r_{\mathrm{SIF} 1}=0.27 ; r_{\mathrm{SIF} 14}=0.28\right.$; $P<0.0001)$ and in adjusted regression analysis, mean coffee intake was positively associated with SIF1 $1_{\text {LED }} 375 \mathrm{~nm}[0.6,0.2]$ $\left(P=8.9 \times 10^{-12}\right)$ and SIF14 2 ED $456 \mathrm{~nm}[0.4,0.8]\left(P=4.4 \times 10^{-12}\right)$. Decaffeinated coffee consumption, however, was not associated with SIF1 LED $375 \mathrm{~nm}[0.6,0.2](r=0.02 ; P=0.53)$ or SIF14 $4_{\text {LED }} 456 \mathrm{~nm}[0.4,0.8](r=0.01 ; P=0.73)$ in either type of analysis, even after restricting the analysis to subjects reporting a mean consumption of $>1$ cup of caffeinated coffee once weekly (Spearman $r_{\mathrm{SIF} 1}=-0.03, P=0.39 ; r_{\mathrm{SIF} 14}=-0.02$, $P=0.57)$.

Tea consumption was associated with $\mathrm{SIF} 1_{\mathrm{LED}} 375 \mathrm{~nm}[0.6,0.2]$ in Spearman correlations $(r=0.06, P=0.04)$ but not in adjusted regression analyses $(P=0.13)$.

Because $84 \%$ of subjects reported consuming regular, caffeinated cola never or less than once per month, we did not use this variable in analyses. However, low-calorie caffeinated cola in Spearman analyses was associated with SIF14 $456 \mathrm{~nm}[0.4,0.8](r=0.08, P=0.006)$ and in covariate adjusted an-

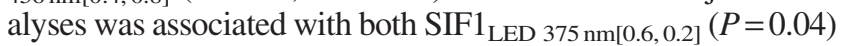
and SIF14 1 LED $456 \mathrm{~nm}[0.4,0.8](P=0.02)$.

Finally, when including both mean coffee intake and mean caffeine consumption in the model, the effect for mean coffee intake disappears $\left(P=0.19\right.$ for $\mathrm{SIF} 1_{\mathrm{LED}} 375 \mathrm{~nm}[0.6,0.2]$ and $P=0.09$ for SIF14 $\mathrm{LED} 456 \mathrm{~nm}[0.4,0.8]$ ), and the positive association for caffeine consumption persists $\left(P=1.9 \times 10^{-5}\right.$ for $\mathrm{SIF}_{\text {LED } 375 \mathrm{~nm}[0.6,0.2]} ; P=1.3 \times 10^{-4}$ for SIF14 ${ }_{\mathrm{LED}} 456 \mathrm{~nm}[0.4,0.8] ; \mathrm{M} 2$ analyses).

\section{Replication in the Pittsburgh EDC study}

Subject characteristics at the time of SIF assessment are shown in Supplementary Table S6. At the time of diet assessment, mean participant age was 31 years with diabetes duration of 22 years. Caffeine was positively associated with SIF $1_{\text {LED }} 375 \mathrm{~nm}[0.6,0.2]$ (Spearman $r=0.31, P<0.0001$ ), similar to the effect size observed in the DCCT/EDIC study, accounting for $9.6 \%$ of the $S I F 1_{\text {LED }} 375 \mathrm{~nm}[0.6,0.2]$ variance in unadjusted analyses. Similar to the effect observed in the DCCT/EDIC study, rs1495741 was associated with $\mathrm{SIF}_{\text {LED } 375 \mathrm{~nm}[0.6,0.2]}(\beta \pm \mathrm{SE},-0.08 \pm 0.03 ; P=0.002)$ and

Table 3. Association of rs1495741 and Caffeine Intake During the Epidemiology of Diabetes Interventions and Complications Study with Skin Intrinsic Fluorescence

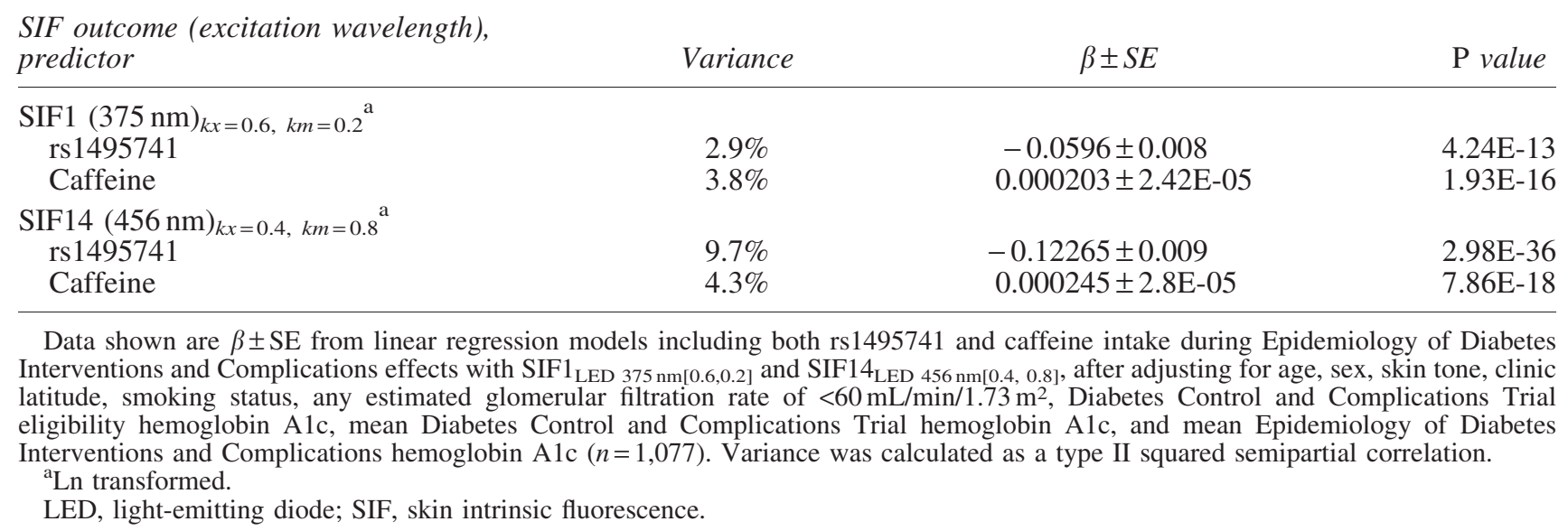


SIF14 ${ }_{\text {LED }} 456 \mathrm{~nm}[0.4,0.8]\left(\beta \pm \mathrm{SE},-0.14 \pm 0.03 ; P=1.5 \times 10^{-5}\right)$, with each copy of the $\mathrm{G}$ allele associated with lower SIF. In adjusted models (Supplementary Table S7), rs1495741 was independently associated with SIF $1_{\text {LED }} 375 \mathrm{~nm}[0.6,0.2]$, but the effect for caffeine intake was attenuated and no longer significant $(P=0.12)$. For SIF14 ${ }_{\text {LED }} 456 \mathrm{~nm}[0.4,0.8]$, however, both rs1495741 and caffeine intake were independently associated with SIF14 LED $456 \mathrm{~nm}[0.4,0.8]$ (Supplementary Table S7).

\section{Discussion}

We recently identified rs1495741, near NAT2, as a major locus for skin fluorescence in subjects with and without diabetes. ${ }^{10}$ Given the role of NAT2 in caffeine metabolism, we examined whether caffeine is associated with SIF, as well as whether the effect of NAT2 (rs1495741) on SIF is independent of caffeine consumption. Here we show that caffeine contributes a proportion of the interindividual variability in SIF measures. Our findings were consistent within the DCCT/EDIC cohort using caffeine data from the DCCT and the EDIC study periods separately and replicated in an independent type 1 diabetes study. Finally, the effect of rs1495741 on SIF appears to be in part independent of caffeine consumption.

To our knowledge, this is the first study to test for an association between caffeine intake and SIF. A previous study of 147 elderly Dutch subjects examining the relationship of dietary AGEs with skin autofluorescence found no significant association between coffee consumption and skin autofluorescence. ${ }^{23}$ Although the excitation level of SIF $1_{\text {LED }}$ $375 \mathrm{~nm}[0.6,0.2]$ is similar to that used by the AGE Reader in the Dutch study (peak excitation, $360 \mathrm{~nm}$ ) there are several reasons that may explain the discrepancy in results between the current study and the former study. First, skin collagen production is altered in the elderly ${ }^{24}$ and therefore may result in no association observed in older subjects. Second, it is not clear how coffee intake was measured by the food habits questionnaire used, as well as whether there was any distinction between caffeinated and decaffeinated coffee consumption. ${ }^{23}$ In the EDIC study, participants reported consumption of both decaffeinated and caffeinated coffee, and we observed a stronger association with SIF when using total caffeine intake versus caffeinated coffee consumption. Finally, The Netherlands has one of the highest consumptions of caffeine in the world, ${ }^{25}$ and coffee intakes were higher in the Dutch study with a mean consumption of 3.4 cups/day, compared with a median of 1 cup of caffeinated coffee daily in the EDIC study. If a nonlinear caffeine-SIF effect exists, whereby there is little association at the upper end of intakes on SIF, then null associations may be observed among high caffeine consumers; however, the range of coffee intake was not stated. ${ }^{23}$

We showed that mean caffeine intake was associated with SIF, independent of age and smoking. Caffeine is positively correlated with age among American adults up to 64 years of age and higher in smokers. ${ }^{26,27}$ Adjusting for both age and smoking attenuated the caffeine-SIF association as they were also positively associated with caffeine intake and SIF in the EDIC study. HbA1c was also shown to contribute to the attenuation in the relationship for mean caffeine intake during EDIC and SIF14 ${ }_{\text {LED }} 456 \mathrm{~nm}[0.4,0.8]$. This was likely due to the positive association of caffeine intake with $\mathrm{HbA} 1 \mathrm{c}$, which may result from caffeine inhibiting glucose uptake by skel- etal muscle and adipocytes, decreasing postprandial glycemic control. ${ }^{28}$ In the DCCT/EDIC study, caffeine consumption independently accounted for $4 \%$ of the variability in SIF (in M2) and, together with rs1495741 and explanatory covariates adjusted for in M2, cumulatively accounted for more than $40 \%$ of the variance in SIF.

Consistent with our study, NAT2 has not been previously associated with coffee or caffeine intake. GWAS of coffee ${ }^{29}$ and caffeine ${ }^{30}$ intake have identified associations for CYP1A2 and AHR, both of which are directly and indirectly involved in caffeine metabolism, respectively. Recently, six additional loci have been implicated in a GWAS of coffee consumption. ${ }^{31}$ Furthermore, a candidate gene approach reported no nominal association of 32 single nucleotdie polymorphisms in NAT2 with caffeine intake using gene-based tests. ${ }^{30}$ On the other hand, NAT2 has been associated with 1-methylxanthine:4-acetamidobutanoate, reflecting metabolites of caffeine metabolism in a GWAS of serum metabolites. ${ }^{13}$ Similarly, a GWAS of urinary metabolite ratios identified a polymorphism in perfect linkage disequilibrium with rs 1495741 to be associated with formate:succinate levels $\left(P=5.1 \times 10^{-16}\right) .{ }^{32}$ Formate may reflect degradation of caffeine because NAT2 metabolizes an intermediate caffeine metabolite and produces 5-acetylamino-6-formylamino-3-methyluracil, ${ }^{12}$ which undergoes deformylation in the urine. ${ }^{33}$

Given that caffeine metabolites are commonly used as a probe for NAT2 acetylation status ${ }^{12,33}$ and that caffeine is associated with SIF, we examined whether the effects of rs1495741 and caffeine are independent. We showed that each was associated with SIF1 $1_{\text {LED }} 375 \mathrm{~nm}[0.6,0.2]$ and SIF14 LED $456 \mathrm{~nm}[0.4,0.8]$ when included in the model together, thus suggesting that the relationship is likely independent. However, because NAT2 was not associated with caffeine intake in the DCCT/EDIC study or in previous studies ${ }^{29-31}$ but instead has been shown to be associated with caffeine metabolite ratios, ${ }^{13,34}$ future studies measuring caffeine metabolites are needed to more concretely examine their independent effects, ${ }^{35}$ as well as establishing whether caffeine metabolites are associated with SIF.

Caffeine readily crosses all biological membranes, ${ }^{25}$ and ingested caffeine has been implicated in protecting against ultraviolet B-induced skin carcinoma in epidemiological and animal studies, partly by protecting the skin as a sunscreen. ${ }^{36,37}$ Caffeine was associated with both $\mathrm{SIF}_{\text {LED } 375 \mathrm{~nm}[0.6,0.2]}$ and SIF14 $4_{\text {LED } 456 \mathrm{~nm}[0.4,0.8]}$, which capture common fluorophores such as AGEs, but also distinct chemicals at each end of the spectrum. ${ }^{2,18}$ Indeed, caffeine was shown to exhibit fluorescent properties with excitation/ emission wavelengths in the ultraviolet range $311 / 363 \mathrm{~nm},{ }^{14}$ but this level was not captured by SIF $1_{\text {LED }} 375 \mathrm{~nm}[0.6,0.2]$, the lowest excitation LED examined in the DCCT/EDIC and the EDC study. Alternatively, the caffeine-SIF association we observed may reflect the effect of caffeine on photolysis of riboflavin, ${ }^{38}$ which has excitation/emission maxima of $450 / 520 \mathrm{~nm}$, corresponding with SIF14 $\operatorname{LED} 456 \mathrm{~nm}[0.4,0.8]{ }^{39}$ Because caffeine can bind to riboflavin and inhibit photolysis, ${ }^{38}$ individuals with high caffeine intake may have higher levels of riboflavin in the skin, resulting in higher skin fluorescence. Finally, we cannot rule out the possibility of the caffeine-SIF association reflecting fluorescence levels of AGEs. We addressed this by adjusting for $\mathrm{HbAlc}$, and although the effect of caffeine on SIF14 ${ }_{\text {LED }} 456 \mathrm{~nm}[0.4,0.8]$ was 
attenuated, it was still significant. Further studies are needed to determine which fluorophore is responsible for the caffeine-SIF association.

Because SIF is positively associated with subclinical atherosclerosis and/or cardiovascular disease mortality, ${ }^{3-7}$ our results suggest that adjusting for caffeine and/or coffee intake may improve prediction estimates for SIF with cardiovascular disease because in moderate amounts coffee consumption may be protective against cardiovascular disease. $^{26,40}$ Similarly, because SIF has been proposed as an alternative noninvasive screening tool in comparison with fasting glucose levels in identifying individuals with prediabetes or type 2 diabetes mellitus, ${ }^{41}$ adjusting for caffeine and/or coffee intake may alter prediction estimates. Components other than caffeine in coffee, such as chlorogenic acid, may be protective against type 2 diabetes mellitus ${ }^{42}$ and AGE formation, ${ }^{43}$ and therefore adjustment for coffee or caffeine as a marker of coffee intake may be useful.

The last measure of caffeine consumption was assessed 4 years prior to SIF in the EDIC study and 8 years prior to SIF in the Pittsburgh EDC study, but despite this, the caffeine-SIF association was robust both within the DCCT/EDIC study and replicated in the EDC study. Food sources contributing to caffeine intake were only available during EDIC years 13-15, and mean intakes of decaffeinated coffee and regular soda consumption were low, somewhat limiting the power of these analyses. Future studies in populations where interindividual intakes for these beverages vary more widely are needed to confirm our observations suggesting that the caffeine-SIF association is due to caffeine and not other components highly correlated with caffeine in coffee or other caffeine food sources. Finally, studies are needed in subjects without diabetes to determine whether the association is diabetes-specific.

In conclusion, our study clearly demonstrates that caffeine is an important contributor to the interindividual variability of SIF measures in people with type 1 diabetes. The association for caffeine intake with SIF appears to be due to caffeine and/or its metabolites rather than other constituents in coffee. Finally, the effect of rs1495741 on SIF is shown to be at least in part independent of caffeine consumption. Accounting for rs1495741 and caffeine intake may alter SIF prediction estimates for risk of cardiovascular disease, prediabetes, and type 2 diabetes mellitus.

\section{Acknowledgments}

We would like to acknowledge the DCCT/EDIC study and Pittsburgh EDC study staff and study participants. In addition, we would like to thank Laura Sampson for her assistance with the FFQ data in the EDIC study. Industry contributors have had no role in the DCCT/EDIC study but have provided free or discounted supplies or equipment to support participants' adherence to the study: Abbott Diabetes Care (Alameda, CA), Animas (Westchester, PA), Bayer Diabetes Care (North America Headquarters, Tarrytown, NY), Becton Dickinson (Franklin Lakes, NJ), Eli Lilly (Indianapolis, IN), Extend Nutrition (St. Louis, MO), Insulet Corporation (Bedford, MA), Lifescan (Milpitas, CA), Medtronic Diabetes (Minneapolis, MN), Nipro Home Diagnostics (Ft. Lauderdale, FL), Nova Diabetes Care (Billerica, MA), Omron (Shelton, CT), Perrigo Diabetes Care (Allegan, MI), Roche Diabetes Care (Indianapolis, IN), and Sanofi-Aventis (Bridgewater, NJ).
The DCCT/EDIC study has been supported by cooperative agreement grants (1982-1993, 2012-2017) and contracts (1982-2012) with the Division of Diabetes Endocrinology and Metabolic Diseases of the National Institute of Diabetes and Digestive and Kidney Disease (current grant numbers U01 DK094176 and U01 DK094157), as well as through support by the National Eye Institute, the National Institute of Neurologic Disorders and Stroke, the General Clinical Research Centers Program (1993-2007), and the Clinical Translational Science Center Program (2006-present), Bethesda, MD.

Additional support for this DCCT/EDIC collaborative study was provided by contract N01-DK-6-2204 from the National Institute of Diabetes and Digestive and Kidney Diseases, grants R01-DK-077510, R01-DK077489, and P60DK20595 from the National Institute of Diabetes and Digestive and Kidney Diseases, and support from Genome Canada through the Ontario Genomics Institute. A.D.P. holds a Canada Research Chair in the Genetics of Complex Diseases. K.M.E. is a recipient of the Gibney Family Scholar Award of the Heart and Stroke Foundation. The Pittsburgh EDC study is supported by grant DK 34818 from the National Institutes of Health.

\section{Author Disclosure Statement}

J.M. is an employee of Veralight. T.J.O. has received research funding from Veralight in the past. K.M.E., R.G.M., T.C., D.M.G., P.A.C., B.H.B., and A.D.P. declare no competing financial interests exist.

\section{References}

1. Hull E, Ediger M, Unione A, et al.: Noninvasive, optical detection of diabetes: model studies with porcine skin. Opt Express 2004;12:4496-4510.

2. Meerwaldt R, Graaff R, Oomen PH, et al.: Simple noninvasive assessment of advanced glycation endproduct accumulation. Diabetologia 2004;47:1324-1330.

3. Lutgers HL, Graaff R, de Vries R, et al.: Carotid artery intima media thickness associates with skin autofluoresence in non-diabetic subjects without clinically manifest cardiovascular disease. Eur J Clin Invest 2010; 40:812-817.

4. Orchard TJ, Lyons TJ, Cleary PA, et al.: The association of skin-intrinsic fluorescence with type 1 diabetes complications in the DCCT/EDIC Study. Diabetes Care 2013;36: 3146-3153.

5. Conway B, Edmundowicz D, Matter N, et al.: Skin fluorescence correlates strongly with coronary artery calcification severity in type 1 diabetes. Diabetes Technol Ther 2010;12:339-345.

6. Meerwaldt R, Lutgers HL, Links TP, et al.: Skin autofluorescence is a strong predictor of cardiac mortality in diabetes. Diabetes Care 2007;30:107-112.

7. Meerwaldt R, Hartog JW, Graaff R, et al.: Skin autofluorescence, a measure of cumulative metabolic stress and advanced glycation end products, predicts mortality in hemodialysis patients. J Am Soc Nephrol 2005;16:36873693.

8. Cleary PA, Braffett BH, Orchard T, et al.: Clinical and technical factors associated with skin intrinsic fluorescence in subjects with type 1 diabetes from the Diabetes Control and Complications Trial/Epidemiology of Diabetes 
Interventions and Complications Study. Diabetes Technol Ther 2013;15:466-474.

9. Garcia-Closas M, Hein DW, Silverman D, et al.: A single nucleotide polymorphism tags variation in the arylamine Nacetyltransferase 2 phenotype in populations of European background. Pharmacogenet Genomics 2011;21:231-236.

10. Eny KM, Lutgers HL, Maynard J, et al.: GWAS identifies a N-acetyltransferase 2 (NAT2) acetylator status tag single nucleotide polymorphism to be a major locus for skin fluorescence. Diabetologia 2014;57:1623-1634.

11. Hein DW, Doll MA, Fretland AJ, et al.: Molecular genetics and epidemiology of the NAT1 and NAT2 acetylation polymorphisms. Cancer Epidemiol Biomarkers Prev 2000;9:29-42.

12. Grant DM, Tang BK, Kalow W: A simple test for acetylator phenotype using caffeine. Br J Clin Pharmacol 1984;17:459-464.

13. Suhre K, Shin SY, Petersen AK, et al.: Human metabolic individuality in biomedical and pharmaceutical research. Nature 2011;477:54-60.

14. Karim MM, Jeon CW, Lee HS, et al.: Simultaneous determination of acetylsalicylic acid and caffeine in pharmaceutical formulation by first derivative synchronous fluorimetric method. J Fluoresc 2006;16:713-721.

15. The Diabetes Control and Complications Trial Research Group: The effect of intensive treatment of diabetes on the development and progression of long-term complications in insulin-dependent diabetes mellitus. $\mathrm{N}$ Engl J Med 1993;329:977-986.

16. Epidemiology of Diabetes Interventions and Complications (EDIC) Research Group: Design, implementation, and preliminary results of a long-term follow-up of the Diabetes Control and Complications Trial cohort. Diabetes Care 1999;22:99-111.

17. Felipe DL, Hempe JM, Liu S, et al.: Skin intrinsic fluorescence is associated with hemoglobin $\mathrm{A}_{1 \mathrm{c}}$ and hemoglobin glycation index but not mean blood glucose in children with type 1 diabetes. Diabetes Care 2011;34:1816-1820.

18. Richards-Kortum R, Sevick-Muraca E: Quantitative optical spectroscopy for tissue diagnosis. Annu Rev Phys Chem 1996;47:555-606.

19. Schmidt LE, Cox MS, Buzzard IM, et al.: Reproducibility of a comprehensive diet history in the Diabetes Control and Complications Trial. The DCCT Research Group. J Am Diet Assoc 1994;94:1392-1397.

20. Willett WC, Sampson L, Stampfer MJ, et al.: Reproducibility and validity of a semiquantitative food frequency questionnaire. Am J Epidemiol 1985;122:51-65.

21. Salvini S, Hunter DJ, Sampson L, et al.: Food-based validation of a dietary questionnaire: the effects of week-toweek variation in food consumption. Int $\mathrm{J}$ Epidemiol 1989;18:858-867.

22. Orchard TJ, Dorman JS, Maser RE, et al.: Prevalence of complications in IDDM by sex and duration. Pittsburgh Epidemiology of Diabetes Complications Study II. Diabetes 1990;39:1116-1124.

23. Jochemsen BM, Mulder DJ, van Doormaal JJ, et al.: Relation between food and drinking habits, and skin autofluorescence and intima media thickness in subjects at high cardiovascular risk. J Food Nutr Res 2009;48:51-58.

24. Varani J, Dame MK, Rittie L, et al.: Decreased collagen production in chronologically aged skin: roles of agedependent alteration in fibroblast function and defective mechanical stimulation. Am J Pathol 2006;168:1861-1868.
25. Fredholm BB, Battig K, Holmen J, et al.: Actions of caffeine in the brain with special reference to factors that contribute to its widespread use. Pharmacol Rev 1999;51:83-133.

26. Freedman ND, Park Y, Abnet CC, et al.: Association of coffee drinking with total and cause-specific mortality. $\mathrm{N}$ Engl J Med 2012;366:1891-1904.

27. Mitchell DC, Knight CA, Hockenberry J, et al.: Beverage caffeine intakes in the U.S. Food Chem Toxicol 2014; 63:136-142.

28. Lane JD, Feinglos MN, Surwit RS: Caffeine increases ambulatory glucose and postprandial responses in coffee drinkers with type 2 diabetes. Diabetes Care 2008;31:221222.

29. Amin N, Byrne E, Johnson J, et al.: Genome-wide association analysis of coffee drinking suggests association with CYP1A1/CYP1A2 and NRCAM. Mol Psychiatry 2012;17: 1116-1129.

30. Cornelis MC, Monda KL, Yu K, et al.: Genome-wide metaanalysis identifies regions on 7p21 (AHR) and 15q24 (CYP1A2) as determinants of habitual caffeine consumption. PLoS Genet 2011;7:e1002033.

31. Cornelis MC, Byrne EM, Esko T, et al.: Genome-wide meta-analysis identifies six novel loci associated with habitual coffee consumption. Mol Psychiatry 2015;20:647656.

32. Suhre K, Wallaschofski H, Raffler J, et al.: A genome-wide association study of metabolic traits in human urine. Nat Genet 2011;43:565-569.

33. Nyeki A, Buclin T, Biollaz J, et al.: NAT2 and CYP1A2 phenotyping with caffeine: head-to-head comparison of AFMU vs. AAMU in the urine metabolite ratios. Br J Clin Pharmacol 2003;55:62-67.

34. Djordjevic N, Carrillo JA, Roh HK, et al.: Comparison of $\mathrm{N}$-acetyltransferase-2 enzyme genotype-phenotype and xanthine oxidase enzyme activity between Swedes and Koreans. J Clin Pharmacol 2012;52:1527-1534.

35. Davey Smith G: Use of genetic markers and gene-diet interactions for interrogating population-level causal influences of diet on health. Genes Nutr 2011;6:27-43.

36. Song F, Qureshi AA, Han J: Increased caffeine intake is associated with reduced risk of basal cell carcinoma of the skin. Cancer Res 2012;72:3282-3289.

37. Conney AH, Lu YP, Lou YR, et al: Mechanisms of caffeine-induced inhibition of UVB carcinogenesis. Front Oncol 2013;3:144.

38. Ahmad I, Ahmed S, Sheraz MA, et al.: Effect of caffeine complexation on the photolysis of riboflavin in aqueous solution: a kinetic study. Chem Pharm Bull (Tokyo) 2009;57:1363-1370.

39. Sikorska E, Gliszczynska-Swiglo A, Insinska-Rak M, et al.: Simultaneous analysis of riboflavin and aromatic amino acids in beer using fluorescence and multivariate calibration methods. Anal Chim Acta 2008;613:207-217.

40. Ding M, Bhupathiraju SN, Satija A, et al.: Long-term coffee consumption and risk of cardiovascular disease: a systematic review and a dose-response meta-analysis of prospective cohort studies. Circulation 2014;129:643659.

41. Olson BP, Matter NI, Ediger MN, et al.: Noninvasive skin fluorescence spectroscopy is comparable to hemoglobin A1c and fasting plasma glucose for detection of abnormal glucose tolerance. J Diabetes Sci Technol 2013;7:9901000 . 
42. Tunnicliffe JM, Eller LK, Reimer RA, et al.: Chlorogenic acid differentially affects postprandial glucose and glucosedependent insulinotropic polypeptide response in rats. Appl Physiol Nutr Metab 2011;36:650-659.

43. Kim J, Jeong IH, Kim CS, et al.: Chlorogenic acid inhibits the formation of advanced glycation end products and associated protein cross-linking. Arch Pharm Res 2011;34: 495-500.
Address correspondence to: Andrew D. Paterson, MD

Program in Genetics \& Genomic Biology Hospital for Sick Children 686 Bay Street, Room 12.9830 Toronto, ON M5G 0A4, Canada

E-mail: andrew.paterson@utoronto.ca 